\title{
A review of methods for mining idea from text
}

\begin{abstract}
Idea mining is a new and interesting field in the areas of information retrieval research. The thoughts of people are helpful to improve strategic decision making. This paper demonstrates the efficient computational methods of idea characterization based concept by extracting the interesting hidden data from unstructured texts which come in many forms and sizes. It may be stored in patents, publications, reports, documents, Internet etc. We briefly discussed a number of successful text mining tools and text classification to extract the idea with a combination of idea mining measures.
\end{abstract}

Keyword: Idea mining; Information retrieval; Text classification; Text mining 